\title{
Reactivity of the cerebral circulation in patients with carotid occlusion
}

\author{
MARTIN M BROWN, JPH WADE, CCR BISHOP*, RW ROSS RUSSELL \\ From the Institute of Neurology, National Hospital for Nervous Diseases, and *Department of Surgery, \\ St Thomas' Hospital, London, UK
}

SUMMARY Cerebral blood flow (CBF) and the response to hypercapnia (cerebral reactivity) have been measured in 41 patients with unilateral or bilateral internal carotid artery occlusion in an attempt to identify those with limited collateral reserve. Normocapnic CBF was within normal limits in the majority of subjects. The response to hypercapnia varied from normal to absent, with impaired reactivity becoming increasingly likely when more than one artery was diseased. In 19 patients with unilateral carotid occlusion, hemisphere reactivity was well preserved in the majority, but was significantly lower on the side of the occlusion (mean $2.9 \% / \mathrm{mm} \mathrm{Hg}$ ) compared to the normal side (mean $3.4 \% / \mathrm{mm} \mathrm{Hg}$ ). Reactivity on the side of the occlusion was further reduced in 15 patients with occlusion and contralateral internal carotid artery stenosis (mean $1.7 \% / \mathrm{mm} \mathrm{Hg}$ ) and was even lower in seven patients with bilateral occlusion (mean $1.1 \% / \mathrm{mm} \mathrm{Hg}$ ). There was no difference in reactivity between asymptomatic hemispheres in the 41 patients (mean $2 \cdot 7 \% / \mathrm{mm} \mathrm{Hg}$ ) and hemispheres in which a previous stroke had occurred (mean $2 \cdot 8 \% / \mathrm{mm} \mathrm{Hg}$ ). In contrast the response in hemispheres subject to continuing transient ischaemic attacks was significantly impaired (mean $1.6 \% / \mathrm{mm} \mathrm{Hg}$ ), suggesting that the cerebral symptoms in some of these patients may have had a haemodynamic origin more often than suspected from the clinical history.

Some patients with occlusion of the internal carotid artery suffer a major stroke, but others present with a minor stroke or a transient ischaemic attack (TIA); occasionally occlusion of one or both carotid arteries occurs without any symptoms. It might be thought that once a carotid artery has occluded completely the risk of further stroke or TIA on the side of the occlusion is over. However, some patients continue to experience delayed vascular events ipsilateral to established carotid occlusion ${ }^{1-8}$ and controversy persists as to the cause of these episodes. Barnett ${ }^{5}$ drew attention to coincidental proximal external carotid and common carotid disease and suggested that this could act as a potential source of emboli to the retina or hemisphere via the collateral circulation. Indeed, haemodynamic factors appeared clinically important in only two of the 27 patients described. Two subsequent studies ${ }^{67}$ have emphasised the importance of coincidental external carotid artery disease. A further potential source of emboli is thrombus within the

Address for reprint requests: Dr MM Brown, Dept of Neurology, The London Hospital, Whitechapel, London E1 1BB, UK.

Received 7 May 1985. Accepted 24 June 1985 stump of the proximal internal carotid with embolisation via anastamotic channels. ${ }^{8}$ On the other hand, some patients undoubtedly have symptoms and signs which point to haemodynamic insufficiency ${ }^{9-12}$ and Sundt and Whisnant ${ }^{1314}$ have suggested that ischaemic symptoms in the region of an occluded artery are usually haemodynamic in origin and therefore an indication for bypass surgery.

Although the brain is supplied by four major arteries and has efficient routes for collateral blood flow, occlusion of one internal carotid artery produces a fall in perfusion pressure in middle cerebral artery branches of the ipsilateral hemisphere. ${ }^{15}$ The initial response is vasodilatation of the small resistance arteries with a consequent fall in vascular resistance and the maintenance of perfusion rates (the phenomenon of autoregulation ${ }^{16}$ ). The degree of vasodilation required to preserve normal blood flow depends on the resistance of the patent cerebral arteries, the circle of Willis and the collateral vessels. When the vascular bed is maximally dilated, any further reduction in mean pressure will lead to a passive fall in blood flow. ${ }^{16}$

Carbon dioxide $\left(\mathrm{CO}_{2}\right)$ is a potent vasodilator of the normal cerebral circulation. ${ }^{17}$ However, if vaso- 
dilatation is already maximal the capacity to increase flow in response to hypercapnia is limited. ${ }^{18}$ The degree of cerebral $\mathrm{CO}_{2}$ reactivity can therefore provide an index of the capacity of the cerebral circulation for further vasodilatation (cerebrovascular reserve). We have measured $\mathrm{CBF}$ and $\mathrm{CO}_{2}$ reactivity in 41 patients with occlusive carotid artery disease in an attempt to identify those with limited cerebrovascular reserve and have related the results to the extent of large vessel disease and to the presence or absence of continuing ischaemic symptoms. Because of the wide range of $\mathrm{CO}_{2}$ reactivity in normal subjects and in those with cerebrovascular disease we have used patients as their own controls by comparing reactivity in the hemisphere on the side of the occluded carotid artery with that of the contralateral hemisphere.

\section{Patients}

Forty-one patients found to have occlusion of one or both internal carotid arteries were studied. The majority of the subjects were ambulant outpatients and none had had a recent major completed stroke. Most subjects were referred by vascular surgeons during the consideration of carotid artery or bypass surgery. They were divided into the following three groups according to the results of recent angiography (digital subtraction venous imaging in the majority). All had patent vertebral arteries.

Unilateral occlusion Nineteen patients with a total occlusion of one internal carotid artery in whom the contralateral internal carotid artery was normal or had minor irregularities only. This group had a mean age of $61 \cdot 1$ (SD 6.6) with a range of 51-75 years.

Occlusion with contralateral stenosis Fifteen patients in whom total occlusion of one internal carotid artery was associated with $50 \%$ or greater stenosis of the internal carotid artery on the contralateral side. This group had a mean age of $64 \cdot 1$ (SD 8.1) with a range of 48-76 years.

Bilateral carotid occlusion Seven patients with total occlusion of both internal carotid arteries. This group had a mean age of $65 \cdot 1(\mathrm{SD} 11 \cdot 7)$ with a range of 41-74 years.

\section{Neurological symptoms}

All the subjects were questioned at the time of the CBF study about neurological symptoms. Seven subjects had no history of previous cerebral or ocular ischaemia. Most of these had been found to have carotid artery occlusion during the course of investigation for cardiac or peripheral vascular disease. The remainder were divided into the following two groups:-

(a) Previous stroke Fourteen patients had a history of previous stroke (including reversible ischaemic neurological deficits) and five a history of previous TIAs, but all had been free of hemisphere symptoms for at least 2 months. The mean age of this group was $60 \cdot 1$ (SD 8.0) years.

(b) Continuing symptoms Twenty patients were classified as having continuing neurological symptoms if they had had symptoms appropriate to the carotid circulation on the side of the occlusion within the previous 2 months. Eight had amaurosis fugax and 12 hemiplegic or hemisensory TIAs. Many patients had frequent attacks; in two the symptoms were clearly associated with change of posture, in three others the association was less certain and in the remainder the attacks occurred at random. The mean age of this group was $63.6(\mathrm{SD} 7 \cdot 9)$ years.

\section{Methods}

CBF was measured by the non-invasive intravenous xenon ${ }^{133}\left(\mathrm{Xe}^{133}\right)$ technique described by Thomas et al. ${ }^{19}$ In brief, a bolus of about $7 \mathrm{mCi}$ of $\mathrm{Xe}^{133}$ dissolved in saline was injected into an arm vein of the recumbant subject and the clearance of the isotope from the cerebral hemispheres monitored by six external $25 \mathrm{~mm}$ diameter scintillation detectors. Expired concentrations of $\mathrm{Xe}^{133}$ were monitored by a 7 th detector and the end tidal levels used to estimate the concentration of recirculating arterial $\mathrm{Xe}^{133}$. Regional $\mathrm{CBF}$ was calculated for each detector from a one minute initial slope analysis of the clearance curves. Results presented in this paper for each hemisphere are means of the initial flow values calculated from three detectors placed over the frontal, fronto-parietal and temporo-parietal regions.

Blood pressure was recorded from the left arm with a standard mercury sphygmomanometer.

Arterial partial pressure of carbon dioxide was estimated from the mean end tidal expired concentration of $\mathrm{CO}_{2}$, monitored throughout the recordings by a Datax CD 300 infrared capnograph.

Each patient had two measurements of CBF within 2 hours separated by at least $\mathbf{4 5}$ minutes to allow for complete elimination of the first dose of $\mathrm{Xe}^{133}$ between measurements. The first CBF was determined with the patient breathing room air (normocapnia) and the second with the patient breathing $5 \% \mathrm{CO}_{2}$ in air (hypercapnia). $\mathrm{CO}_{2}$ was included in the breathing circuit for at least 2 minutes before the $\mathrm{CBF}$

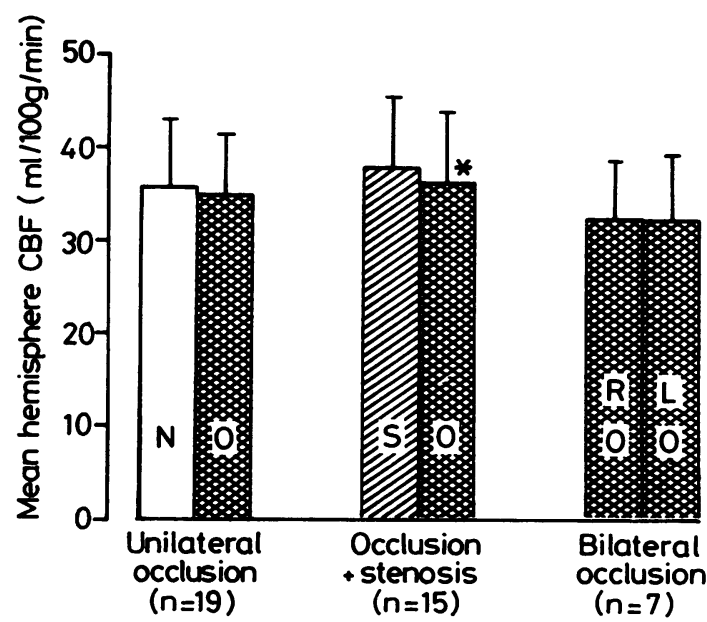

Fig 1 Mean normocapnic hemisphere cerebral blood flow $(C B F) \pm S D$ in three groups of patients related to the state of the ipsilateral carotid artery $(N=$ normal, $O=$ occluded, $S=$ stenosed, $R=$ right, $L=$ left). ${ }^{*} p<0.01$ (paired $t$ test). 
measurement to allow for stabilisation of end-tidal $\mathrm{CO}_{2}$ concentrations, and was continued throughout the $\mathrm{CBF}$ measurement. This technique produced an average sustained rise of $11 \mathrm{~mm} \mathrm{Hg}(1.5 \mathrm{kPa})$ in arterial $\mathrm{pCO}_{2}$ between measurements.

Hemisphere $\mathrm{CO}_{2}$ reactivity was calculated as the percentage rise in $\mathrm{CBF}$ above normocapnic values per $\mathrm{mm} \mathrm{Hg}$ increase in $\mathrm{pCO}_{2}(1 \mathrm{~mm} \mathrm{Hg}=0.13 \mathrm{kPa})$.

\section{Results}

Figure 1 illustrates mean hemisphere CBF during normocapnia according to the patency of the ipsilateral carotid artery in each group. Mean hemisphere CBF was similar in all three groups. There was a tendency for CBF to be lower on the side of an occlusion compared with the contralateral hemisphere, but the difference was small and only significant in the group with contralateral stenosis (mean difference $1.6 \mathrm{ml} / 100 \mathrm{~g} / \mathrm{min}$, paired $t=2.88$ $\mathrm{p}<0.02$ ). Mean CBF was also slightly lower in the patients with bilateral occlusion compared with the other two groups but the difference was not significant (mean $3.7 \mathrm{ml} / 100 \mathrm{~g} / \mathrm{min}$ lower than patent hemispheres, Student's $t=1 \cdot 47$ ).

Figure 2 shows the mean response to hypercapnia in each group and fig 3 shows the resulting values for mean hemisphere $\mathrm{CO}_{2}$ reactivity. In patients with unilateral occlusion hemisphere reactivity was normal on the side of the patent carotid artery (mean $3.4 \% / \mathrm{mm} \mathrm{Hg}$ ), but was significantly reduced on the side of the occlusion (mean $2.9 \% / \mathrm{mm} \mathrm{Hg}$, paired $t=2.41, \mathrm{p}<0.05)$. In the patients with occlusion and contralateral stenosis mean reactivity was lower in both hemispheres and again was significantly reduced on the side of the occlusion (mean $1.7 \% / \mathrm{mm} \mathrm{Hg}$ ) compared with the contralateral hemisphere (mean $2.6 \% / \mathrm{mm} \mathrm{Hg}$, Student's $t=3.18 \mathrm{p}<0.01$ ). This impaired response to hypercapnia on the side of the occlusion in patients with contralateral stenosis was significantly lower than that of the normal hemisphere (Student's $t=3.33, \mathrm{p}<0.01$ ) and occluded hemispheres of the patients with unilateral occlusion (Student's $t=2.56, \mathrm{p}<0.02$ ). The patients with

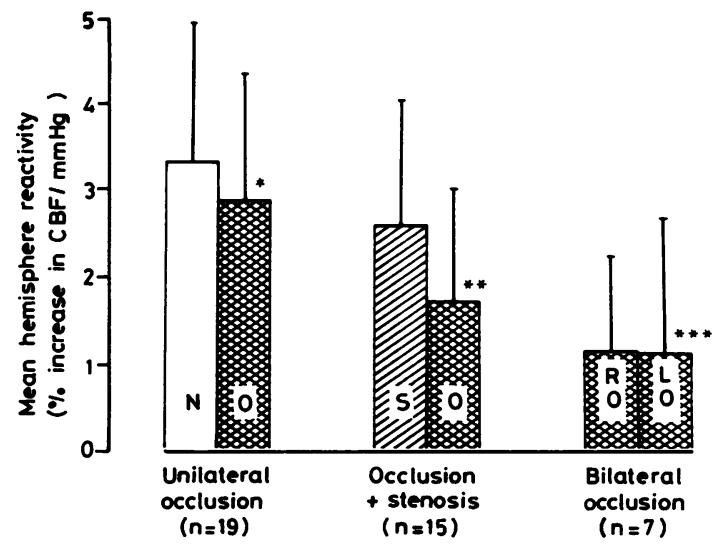

Fig 3 Mean hemisphere carbon dioxide reactivity ( $\pm S D$ ) in three groups of patients related to the state of the ipsilateral carotid artery ( $N=$ normal, $O=$ occluded, $S=$ stenosed, $R=$ right, $L=$ left $) .{ }^{*},{ }^{* *},{ }^{* *} p=<0.05$, $<0.01,<0.001$ (Student's t test, occluded vs normal).

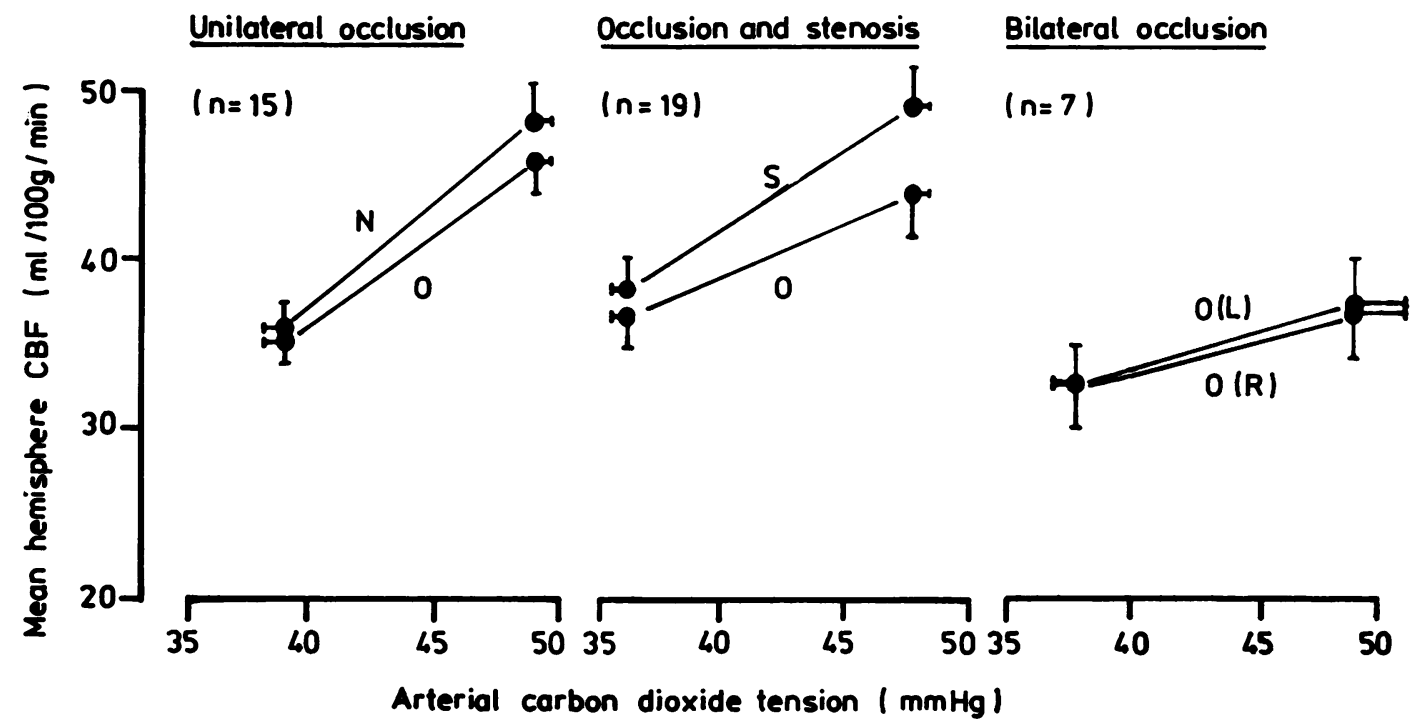

Fig 2 Effect of hypercapnia on cerebral blood flow (CBF) $\pm S E M$ in three groups of patients related to the state of the ipsilateral carotid artery $(N=$ normal, $O=$ occluded, $S=$ stenosed, $R=$ right, $L=l e f t)$. 


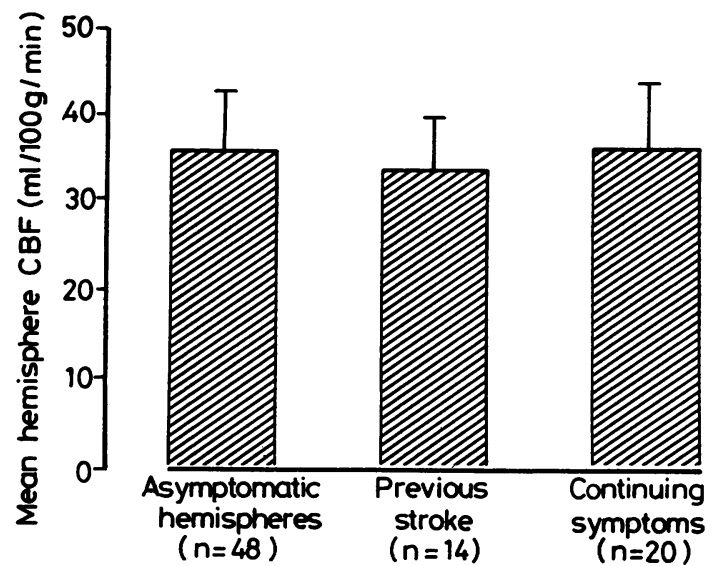

Fig 4 Mean normocapnic hemisphere cerebral blood flow $(C B F) \pm S D$ in patients with carotid occlusion related to ipsilateral symptoms.

bilateral carotid artery occlusion had even greater impairment of the response to hypercapnia with mean hemisphere reactivity of only $1 \cdot 1 \% / \mathrm{mm} \mathrm{Hg}$, which was also highly significantly lower than that of the normal hemispheres (Student's $t=8.94, \mathrm{p}<0.001$ ) and occluded hemispheres of the patients with unilateral occlusion (Student's $t=8.08, \mathrm{p}<0.001$ ). Figure 2 illustrates that the rise in $\mathrm{pCO}_{2}$ was similar in each group and the results could not be explained by changes in blood pressure (BP) which rose during hypercapnia in the majority of subjects (mean normocapnic BP 167/91; mean hypercapnic BP 183/99).

In all groups there was a wide range in hemisphere reactivity from normal to absent responses, but impaired reactivity on the side of an occlusion became increasingly likely with increasing severity of contralateral carotid artery disease. For example, figures of less than $1.5 \% / \mathrm{mm} \mathrm{Hg}$ were found on the side of the occlusion in $11 \%$ of patients with unilateral occlusion, $60 \%$ of patients with occlusion and contralateral stenosis and in at least one hemisphere in $86 \%$ of the patients with bilateral occlusion.

Figures 4 and 5 illustrate the relationships between mean normocapnic $\mathrm{CBF}$, hemisphere reactivity and the patients' symptoms. There was no significant difference between normocapnic CBF in asymptomatic hemispheres, hemispheres with a history of previous ipsilateral stroke or hemispheres with continuing ipsilateral symptoms. There was also no difference in mean $\mathrm{CO}_{2}$ reactivity between asymptomatic hemispheres and hemispheres with a previous stroke. However, reactivity in hemispheres subject to continuing symptoms was significantly impaired compared with both asymptomatic hemispheres (Student's $t=2.76, \mathrm{p}<0.01$ ) and hemispheres with a previous stroke (Student's $t=2.59, \mathrm{p}<0.02$ ). This contrast was most striking in the patients with bilateral occlusion, among whom patients with continuing symptoms had a mean cerebral reactivity of only $0.4 \% / \mathrm{mm} \mathrm{Hg}$ compared with a mean of $2 \% / \mathrm{mm} \mathrm{Hg}$ in those not currently symptomatic. The individual reactivity values on the side of an occlusion are illustrated in fig 6 for each group according to whether the patient had continuing ipsilateral symptoms or not. Fifty-five per cent of patients with continuing symptoms had hemisphere reactivities of less than $1.5 \% / \mathrm{mm} \mathrm{Hg}$, compared with only $19 \%$ of asymptomatic or previous stroke patients. Of the two

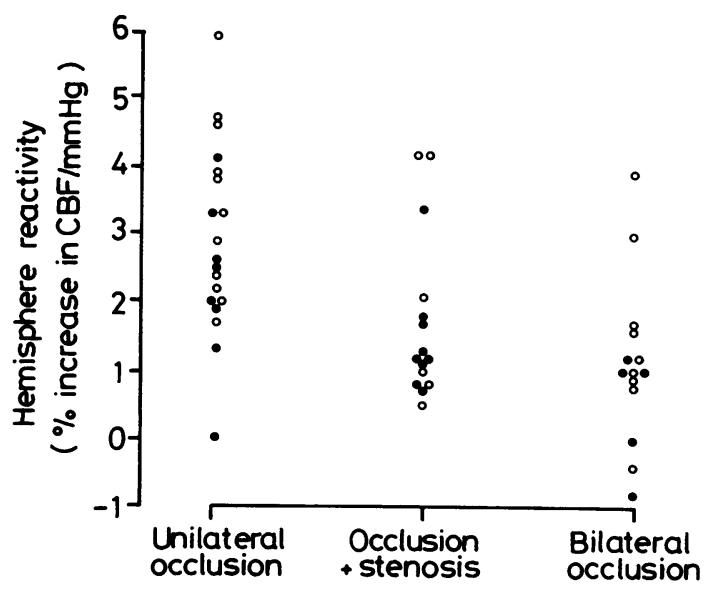

Fig 6 Individual hemisphere carbon dioxide reactivity on the side of the occluded carotid artery in three groups of patients related to ipsilateral symptoms (closed circles = continued symptoms, open circles = asymptomatic or previous stroke).

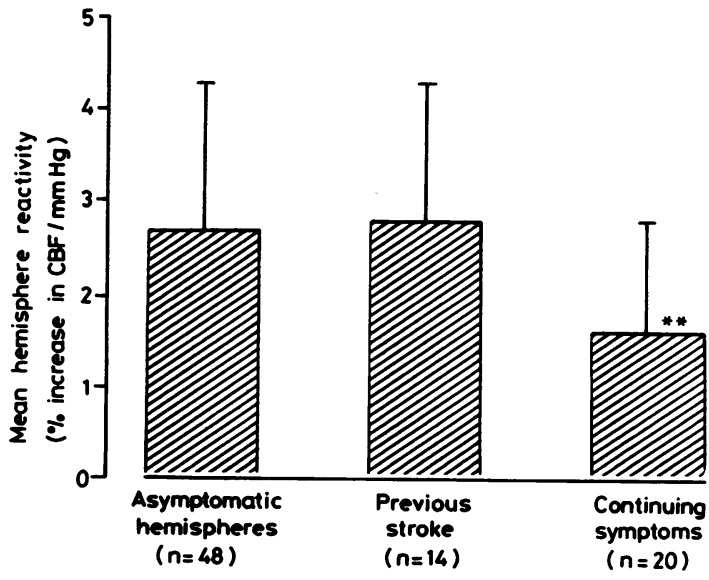

Fig 5 Mean hemisphere carbon dioxide reactivity $( \pm S D$ ) in patients with carotid occlusion related to ipsilateral symptoms. ${ }^{* *} p=<0.01$ (Student's $t$ test, continuing symptoms vs asymptomatic). 
patients in whom TIAs were clearly precipitated by changes in posture, one had the lowest reactivity encountered (CBF actually falling by $0.8 \% / \mathrm{mm} \mathrm{Hg}$ during hypercapnia) and the other had a reactivity of only $1.2 \% / \mathrm{mm} \mathrm{Hg}$. Comparing individual hemispheres, it was found that an asymmetrical reduction in the symptomatic hemisphere compared to the contralateral hemisphere was even more closely associated with continuing symptoms. Reactivity on the ipsilateral side was less than $75 \%$ of the contralateral hemisphere in $65 \%$ of patients with continuing symptoms but in only $3(14 \%)$ patients who were asymptomatic or had previous stroke.

\section{Discussion}

These studies demonstrate that at normal levels of arterial $\mathrm{pCO}_{2}$ autoregulatory mechanisms are able to maintain nearly normal resting CBF in the presence of unilateral carotid occlusion, which is in accord with previous studies. ${ }^{20-25}$ There was a tendency for hemisphere CBF to be slightly lower on the side of the occlusion compared with the other hemisphere but the difference was only significant if the contralateral artery was stenosed. In contrast, when the collateral reserve was tested by $\mathrm{CO}_{2}$ inhalation an impaired CBF response was revealed on the side of the occlusion in many patients. Such impaired $\mathrm{CO}_{2}$ reactivity became increasingly likely when more than one carotid artery was diseased.

The response to hypercapnia on the side of the normal carotid artery in patients with unilateral occlusion was preserved with a mean reactivity of $3.4 \% / \mathrm{mm} \mathrm{Hg}$, which is close to the figure of $3.1 \% / \mathrm{mm} \mathrm{Hg}$ found in a group of patients with cerebrovascular symptoms but normal carotid arteries by Bullock et al, ${ }^{25}$ using a similar method. In comparison with these normal hemispheres $\mathrm{CO}_{2}$ reactivity was significantly lower on the side of the occlusion (mean $2.9 \% / \mathrm{mm} \mathrm{Hg}$ ) in the patients with unilateral occlusion, but was still adequately preserved in many patients. Impaired reactivity was more common in the patients in whom carotid occlusion was associated with contralateral carotid stenosis and the mean reactivity was more significantly reduced on the occluded side at $1.7 \% / \mathrm{mm} \mathrm{Hg}$. The most striking abnormalities were found in the patients with bilateral occlusion. Although this group had a normocapnic CBF which was only slightly lower than normal, $\mathrm{CO}_{2}$ reactivity was grossly impaired bilaterally in all but one patient and the mean reactivity of $1.1 \% / \mathrm{mm} \mathrm{Hg}$ was lower than in the other two groups. These results confirm findings from earlier studies where compromised reactivity has been related to the extent of contralateral arterial disease. ${ }^{22} 2325$
A failure to respond to hypercapnia implies either insensitivity to the metabolic stimulus or that the capacity to increase CBF is limited because vasodilatation is already maximum. The latter explanation has been suggested by previous studies. ${ }^{20-22 ~ 24-27}$ It has also been invoked as the mechanism behind the loss of $\mathrm{CO}_{2}$ reactivity which occurs under conditions of severe hypotension ${ }^{18}$ and may also explain loss of reactivity in the vicinity of acute infarction. ${ }^{28} \mathrm{We}$ suggest that our finding of a graduated reduction in reactivity with increasing severity of carotid artery disease reflects increasing distal vasodilation. This conclusion agrees well with the studies of Gibbs et al, ${ }^{29}$ who reported an increase in cerebral blood volume distal to carotid occlusion, reflecting focal dilatation, which was greatest in the patients with severest arterial disease. It is also consistent with the finding of Norrving $e t a^{23}$ that impaired reactivity in patients with carotid occlusion correlated with angiographic evidence of inadequate collateral supply.

More intriguing than the morphological correlate is our finding that there was a close association between continuing TIAs and a loss of responsiveness to $\mathrm{CO}_{2}$. The present data suggest that continuing cerebral symptoms after carotid occlusion may have a haemodynamic origin more often than suspected from the history since TIAs were clearly related to change of posture in only two of our 20 subjects with recurrent symptoms. However, our study was based on a highly selected group of patients and it remains uncertain how often haemodynamic factors are important in the majority of patients with delayed ischaemic events.

On superficial analysis, surgical revascularisation would appear to be a logical treatment for patients with haemodynamic symptoms or impaired cerebrovascular reserve. Baron et al, ${ }^{11}$ in a detailed study of one patient with posturally induced hemispheric symptoms, reported a dramatic clinical improvement with concomitant normalisation of measurements of critical perfusion, following a successful extracranial/ intracranial $(\mathrm{EC} / \mathrm{IC})$ bypass. On the other hand, even if a subgroup of patients with haemodynamic TIA can be identified it does not necessarily follow that surgical revascularisation is indicated because the symptoms may resolve with time ${ }^{30}$ presumably as collaterals enlarge. Furthermore, there is recent evidence that in general EC/IC bypass does not protect against further stroke in patients with internal carotid occlusion (EC/IC Bypass Study Group ${ }^{31}$ preliminary results). However, the protocol of the EC/IC study group ${ }^{32}$ did not request measurements of cerebral blood flow, nor was there any other systematic attempt to differentiate between embolic and haemodynamic TIA. The present study has demonstrated that cerebrovascular reserve is likely to 
be reduced in patients with recurrent TIAs and multiple vessel disease. It should prove possible to use the EC/IC data base to determine if surgery reduces the chance of stroke in this particular sub-group.

We are very grateful to Professor N Browse, Professor J Marshall, Miss A Mansfield, Dr DJ Thomas, Dr R Frackowiak, Dr MJ Harrison, Dr J Gawler, Dr C Clarke, Dr A Gale and Professor PK Thomas for referring patients. Mr E Zilkha and Miss S Redmond assisted with the CBF measurements.

\section{References}

${ }^{1}$ McDowell FH, Potes J, Groch S. The natural history of internal carotid and vertebral-basilar artery occlusion. Neurology (Minneap) 1961;11:153-7.

${ }^{2}$ Marshall J. The management of occlusion and stenosis of the internal carotid artery. Neurology (Minneap) 1966;16:1087-93.

${ }^{3}$ Dyken ML, Klatte E, Kolar OJ, et al. Complete occlusion of common or internal carotid arteries. Arch Neurol 1974;30:343-6.

${ }^{4}$ Fields WS, Lemak NA. Joint study of extracranial arterial occlusion X. Internal carotid artery occlusion. JAMA 1976;235:2734-8.

${ }^{5}$ Barnett HJM. Delayed cerebral ischaemic episodes distal to occlusion of major cerebral arteries. Neurology (Minneap) 1978;28:769-74.

${ }^{6}$ Countee RW, Vijayanathian T. External carotid artery in internal carotid artery occlusion. Angiographic, therapeutic and prognostic considerations. Stroke 1979;10:450-60.

${ }^{7}$ Bogousslavsky J, Regli F, Hungerbuhler JP, Chrzanowski $R$. Transient ischaemic attacks and external carotid artery: retrospective study of 23 patients with an occlusion of the internal carotid artery. Stroke 1981;12:627-30.

${ }^{8}$ Barnett HJM, Peerless SJ, Kaufmann JCE. "Stump" of the internal carotid artery-a source for further cerebral embolic ischaemia. Stroke 1978;9:448-56.

${ }^{9}$ Sundt TM, Siekert RG, Piepgras DG, Sharbrough FW, Houser OW. Bypass surgery for vascular disease of the carotid system. Mayo Clin Proc 1976;51:677-92.

${ }^{10}$ Russell RWR, Page NRG. Critical perfusion of brain and retina. Brain 1980;106:419-34.

${ }^{11}$ Baron JC, Bousser MG, Rey A, Guilard A, Comar D, Castaigne P. Reversal of focal "misery-perfusion syndrome" by extra-intracranial bypass in hemodynamic cerebral ischaemia. Stroke 1981;12:454-9.

12 Stark RJ, Wodak J. Primary orthostatic cerebral ischaemia. J Neurol Neurosurg Psychiatry 1983;46: 883-91.

${ }^{13}$ Sundt TM, Whisnant JP, Fode NC, Piepgras DG, Houser OW. Results, complications and follow-up of $415 \mathrm{By}-$ pass operations for occlusive disease of the carotid system. Mayo Clin Proc 1985;60:230-40.

14 Whisnant JP, Sundt TM, Fode NC. Long-term mortality and stroke morbidity after superficial temporal arterymiddle cerebral artery bypass operation. Mayo Clin Proc 1985;60:241-6.
${ }^{15}$ Yonekawa Y, Yamagata S, Moritake K, Taki W, Handa $H$. Intraoperative intraarterial pressure measurement in the cerebrovascular occlusive disease. In: Gotch F, Nagai H, Tazaki Y, eds. Acta Neurol Scand 1979;60 (Supp 72):506-7.

${ }^{16}$ Harper AM. Autoregulation of cerebral blood flow: influence of the arterial blood pressure on the blood flow through the cerebral cortex. $J$ Neurol Neurosurg Psychiatry 1966;29:398-403.

${ }^{17}$ Olesen J, Paulson OB, Lassen NA. Regional cerebral blood flow in man determined by the initial slope of the clearance curve of intraarterially injected $133 \mathrm{Xe}$. Stroke 1971;2:519-40.

${ }^{18}$ Harper AM, Glass HI. Effect of alterations in the arterial carbon dioxide tension on the blood flow through the cerebral cortex at normal and low arterial pressures. $J$ Neurol Neurosurg Psychiatry 1965;28:449-52.

19 Thomas DJ, Zilkha E, Redmond S, et al. An intravenous 133 xenon clearance technique for measuring cerebral blood flow. J Neurol Sci 1979;40:53-63.

${ }^{20}$ Dyken M, Campbell RL, Frayser R. Cerebral blood flow, oxygen utilisation and vascular reactivity. Neurology (Minneap) 1970;20:1127-32.

${ }^{21}$ Dyken ML. Intracranial "steal" in complete occlusion of the internal carotid artery: variations in response to $5 \%$ carbon dioxide and $100 \%$ oxygen. Eur Neurol 1971-1972;6:301-5.

${ }^{22}$ Takagi Y, Hata T, Ishitobi K, Kitagawa Y. Cerebral blood flow and $\mathrm{CO} 2$ reactivity before and after carotid endarterectomy. In: Gotoh F, Nagai H, Tazaki Y, eds. Acta Neurol Scand 1979;60 (Suppl 72):506-7.

${ }^{23}$ Norrving B, Nilsson B, Risberg J. rCBF in patients with carotid occlusion: resting and hypercapnic flow related to collateral pattern. Stroke 1982;13:155-62.

${ }^{24}$ Halsey JH, Morawetz RB, Blauenstein UW. The hemodynamic effect of STA-MCA bypass. Stroke 1982; 13:163-7.

${ }^{25}$ Bullock R, Mendelow AD, Bone I, Patterson J, Macleod J, Allardice G. Cerebral blood flow and $\mathrm{CO} 2$ responsivess as an indicator of collateral reserve capacity in patients with carotid arterial disease. $B r J$ Surg 1985;72:348-51.

${ }^{26}$ Novack P, Shenkin HA, Bortin L, Goluboff B, Soffe AM. The effects of carbon dioxide inhalation upon the cerebral blood flow and cerebral oxygen consumption in vascular disease. J Clin Invest 1953;32:696-702.

${ }^{27}$ Fazekas JF, Alman RW. Maximal dilatation of cerebral vessels. Arch Neurol 1964;11:303-9.

${ }^{28}$ Hoedt-Rasmussen K, Skinhoj E, Paulson O, et al. Regional cerebral blood flow in acute apoplexy. Arch Neurol 1967;17:271-81.

${ }^{29}$ Gibbs JM, Wise RJS, Leenders KL, Jones T. Evaluation of cerebral perfusion reserve in patients with carotid artery occlusion. Lancet 1984;1:310-4.

${ }^{30}$ Caplan LR, Sergay S. Positional cerebral ischaemia. $J$ Neurol Neurosurg Psychiatry 1976;39:385-91.

${ }^{31}$ EC/IC Bypass Study Group. Failure of EC/IC arterial bypass to reduce the risk of ischemic stroke. $N$ Engl J Med 1985;313:1191-2000.

${ }^{32}$ The EC/IC Bypass Study Group. The international cooperative study of extra/intracranial artery anastamosis (EC/IC Bypass Study): Methodology and entry characteristics. Stroke 1985;16:397-406. 